\title{
Quantitative Measurement of the Optical Cross Sections of Single Nano-objects by Correlative Transmission and Scattering Microspectroscopy
}

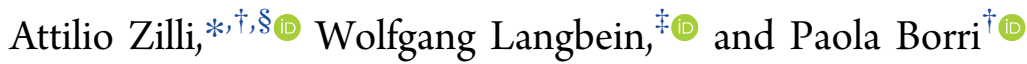 \\ ${ }^{\dagger}$ Cardiff University, School of Biosciences, Museum Avenue, Cardiff CF10 3AX, U.K. \\ ${ }^{\ddagger}$ Cardiff University, School of Physics and Astronomy, The Parade, Cardiff CF24 3AA, U.K.
}

Supporting Information

ABSTRACT: The scattering and absorption of light by nano-objects is a key physical property exploited in many applications, including biosensing and photovoltaics. Yet, its quantification at the single object level is challenging and often requires expensive and complicated techniques. We report a method based on a commercial transmission microscope to measure the optical scattering and absorption cross sections of individual nano-objects. The method applies to microspectroscopy and wide-field image analysis, offering fine spectral information and high throughput sample characterization. Accurate cross-section determination requires detailed modeling of the measurement, which we develop, accounting for

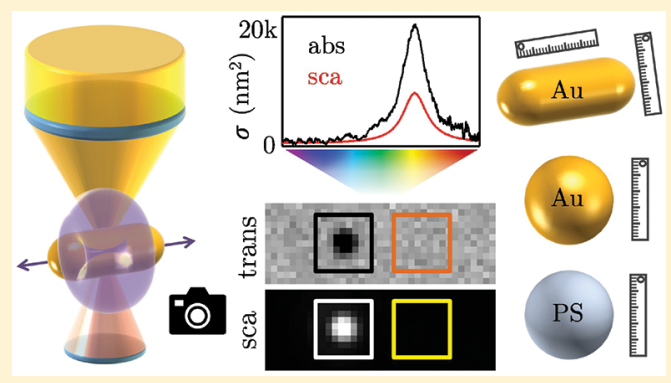
the geometry of the illumination and detection as well as for the presence of a sample substrate. We demonstrate the method on three model systems (gold spheres, gold rods, and polystyrene spheres), which include metallic and dielectric particles, spherical and elongated, placed in a homogeneous medium or on a dielectric substrate. Furthermore, by comparing the measured cross sections with numerical simulations, we are able to determine structural parameters of the studied system, such as the particle diameter and aspect ratio. Our method therefore holds the potential to complement electron microscopy as a simpler and cost-effective tool for structural characterization of single nanoobjects.

KEYWORDS: Nanoparticle, Nanoplasmonics, Rayleigh scattering, Optical cross sections, Particle sizing, Single-particle microscopy, Dark-field microspectroscopy, Absorption microspectroscopy

S cattering and absorption of light by small particles underpin common natural phenomena, such as the colors of the sky and the clouds, the hues of rainbows and irises, and the opalescence of some precious stones. ${ }^{1}$ In particular, localized surface plasmon resonances (LSPRs) enhance the interaction of metal nano-objects (NanOs) with light, originating rich phenomenology and opportunities for concentrating and harnessing electromagnetic energy at the nanoscale. $^{2}$ Numerous applications exploiting the optical properties of metal NanOs have been proposed in fields as diverse as nanomedicine ${ }^{3}$ (e.g., as photothermal targets or labels for optical microscopy), chemical and biological sensing, ${ }^{4}$ and photovoltaics. ${ }^{5}$ The interaction strength of a $\mathrm{NanO}$ with electromagnetic radiation is quantified by its optical cross sections, $\sigma$, defined as the power $P$ removed from an incident plane wave through a given optical process and normalized to the incident intensity, $I_{\mathrm{i}}$, that is, $\sigma \equiv P / I_{\mathrm{i}}$, which corresponds to an effective area of interaction. In the common scenario where inelastic scattering and nonlinear processes are negligible, the optical response is dominated by the cross sections of elastic scattering $\left(\sigma_{\text {sca }}\right)$ and absorption $\left(\sigma_{\text {abs }}\right)$.

Beginning with the invention of the dark-field (DF) "ultramicroscope" more than a century ago, ${ }^{6}$ several exper- imental schemes have been devised to probe $\sigma_{\text {sca }}$ and $\sigma_{\text {abs }}$ at the single-NanO level, in order to avoid effects of sample dispersity and investigate the often sensitive dependence of the optical properties on the geometry. Nonetheless, only in the last two decades methods capable of measuring the magnitude of the cross-section of single-NanOs have emerged. ${ }^{7,8}$ Presently, a prominent quantitative technique is spatial modulation spectroscopy (SMS), ${ }^{9}$ which determines the extinction crosssection, $\sigma_{\mathrm{ext}} \equiv \sigma_{\mathrm{sca}}+\sigma_{\mathrm{abs}}$. Conventional SMS, however, is unable to separate $\sigma_{\mathrm{sca}}$ and $\sigma_{\mathrm{abs}}$ and, in fact, by neglecting the portion of scattering detected, is only accurate for NanOs dominated by absorption. ${ }^{7}$ Photothermal heterodyne imaging (PHI) selectively measures $\sigma_{\text {abs }}$ instead, ${ }^{10}$ but a precise knowledge of the thermal properties of the environment is required for quantitative measurements. As for scattering of single NanOs, just a handful of works in literature report measurements of the $\sigma_{\text {sca }}$ amplitude in absolute units. An interferometric implementation of the SMS principle proposed by Husnik et al., ${ }^{11,12}$ whereby the excitation is split into a laterally displaced signal and a reference beam scanned across

Received: May 19, 2019

Published: July 21, 2019 


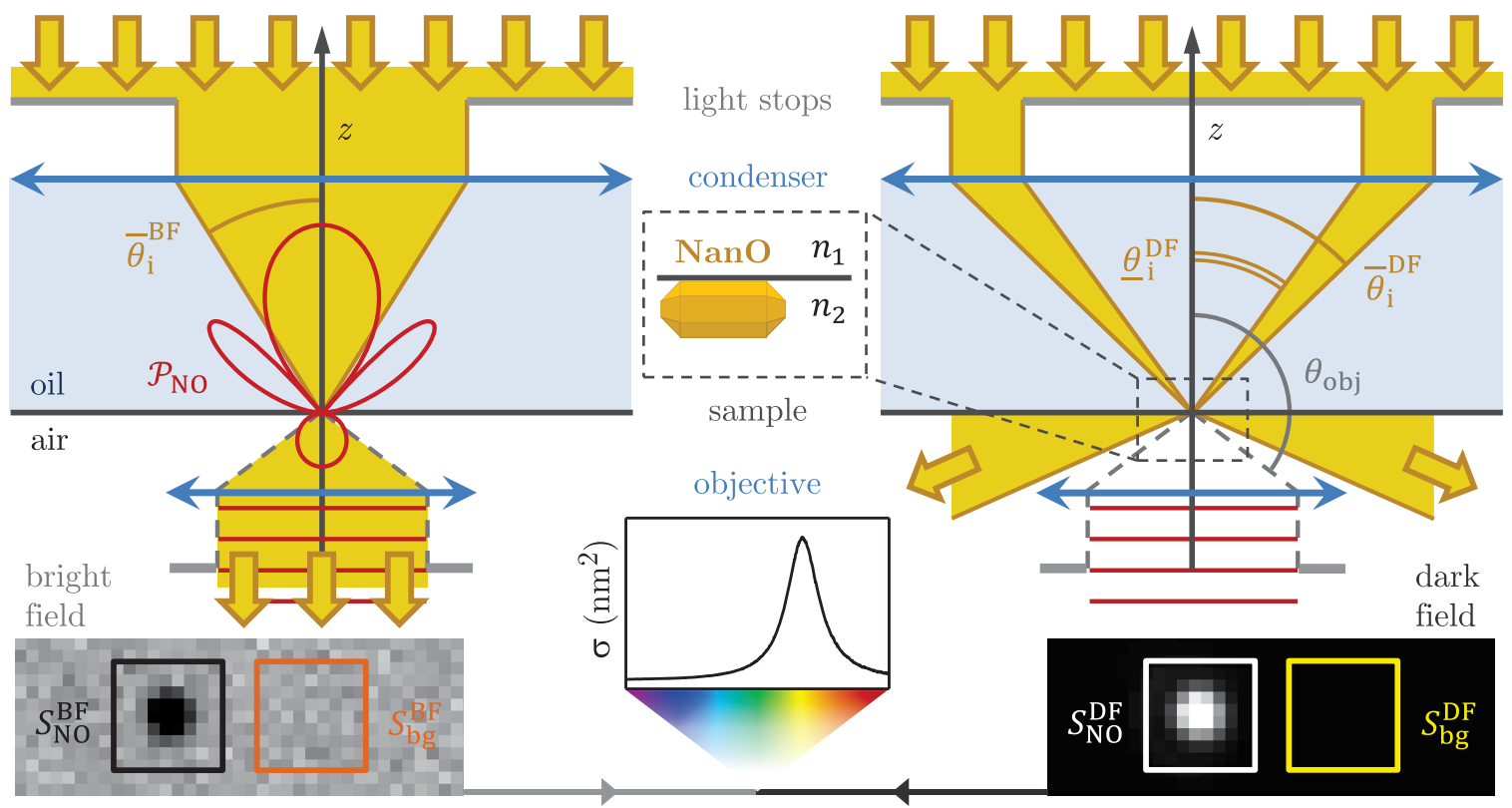

Figure 1. Schematics of the experimental setup for bright-field (BF, left) and dark-field (DF, right) illumination; a detailed description is provided in the text. The illumination angles depicted correspond to $\mathrm{NA}_{\mathrm{i}}^{\mathrm{BF}} \in[0,0.8]$ and $\mathrm{NA}_{\mathrm{i}}^{\mathrm{DF}} \in[0.9,1.1]$; as a consequence, part of the $\mathrm{DF}$ illumination undergoes total internal reflection. The scattering distribution, $\mathcal{P}_{\mathrm{NO}}(\theta)$, is computed in the electrostatic approximation for an elongated NanO placed on a glass/air interface $\left(n_{1}=1.52, n_{2}=1.00\right)$ oriented as in the enlarged image. The image on the bottom left is an example of BF transmission with the $\mathrm{NanO}$ appearing dark on a bright background. The image on the bottom right is an example of DF contrast, with the NanO appearing bright on a dark background. The plot in the bottom middle exemplifies a cross-section spectrum obtained from the BF and DF images.

the sample, can measure separately $\sigma_{\mathrm{sca}}$ and $\sigma_{\mathrm{abs}}$ of large antennas $(\simeq 200 \mathrm{~nm})$. SMS can also operate in a spectroscopic fashion using a broadband source; Pellarin et al. ${ }^{13}$ show a single example of quantitative $\sigma_{\text {sca }}$ and $\sigma_{\text {abs }}$ measurement on a large $(\simeq 200 \mathrm{~nm})$ scattering-dominated silver cube dimer. The separation the $\sigma_{\text {ext }}$ signal into the $\sigma_{\text {sca }}$ and $\sigma_{\text {abs }}$ contributions relies on simulating numerically the optical properties of each investigated $\mathrm{NanO}$ based on its specific geometry measured with transmission electron microscopy (TEM). Finally, interferometric scattering microscopy (iSCAT) ${ }^{14}$ deserves a mention as a scattering-based imaging approach, which proved extremely sensitive for detecting small scatterers such as individual biological macromolecules. ${ }^{15}$ Albeit to the best of our knowledge iSCAT has not yet been used to directly measure the magnitude of $\sigma_{\mathrm{sca}}$ the signal intensity as a function of the focus position can be computed, either analytically in the electrostatic approximation ${ }^{16}$ or numerically, ${ }^{17}$ and quantitatively compared to the experiment.

All the aforementioned techniques require expensive experimental equipment, such as lasers, modulating elements, and lock-in amplifiers, as well as complex analysis procedures, often based on a precise knowledge of the exciting point spread function (PSF). These demands have prevented widespread adoption of these approaches, and as a consequence, most single-NanO studies to-date rely on simpler, nonquantitative approaches such as DF microspectroscopy (possibly with some calibration $^{18,19}$ ) and thereby limit themselves to spectral properties such as position, width, and polarization of LSPRs in metal NanOs. Yet the absolute values of $\sigma_{\text {sca }}$ and $\sigma_{\text {abs }}$ are important for all applications whose performance relies on a strong (or weak) scattering or absorption. ${ }^{3-5}$ This highlights an unmet need for an easy-to-implement and use quantitative experimental tool.

In this work, we present a method that requires just a commercial optical microscope equipped with an incoherent light source and an imaging array (optionally coupled to a spectrometer to obtain detailed spectral information) and demonstrate its accuracy when applied to model systems of diverse materials, shapes, and local environments. The paper is organized as follows: in the Quantitative Method section, we describe the quantitative analysis that enables us to retrieve the cross-section magnitude from the spectroscopy or imaging data. In the Experimental Results section, we apply the method to three technologically relevant NanOs: gold spheres, gold rods, and polystyrene spheres. The accuracy of our results is examined in the Discussion and Conclusions section.

\section{QUANTITATIVE METHOD}

Correlating Transmission and Scattering. The geometry of the experimental setup considered is sketched in Figure 1. Broadband illumination is provided by an incoherent source and focused by a high numerical aperture (NA) oil-immersion condenser lens on the sample plane, where the NanOs are immobilized on a transparent substrate. The NanOs are imaged onto the sensor of choice by an objective of $\left[\theta_{\mathrm{obj}}, \pi\right]$ detection polar angle range determined by $\mathrm{NA}_{\mathrm{obj}} \equiv n_{2} \sin \theta_{\mathrm{obj}}$. The polar angle range of illumination is defined instead by light stops placed in the back focal plane (BFP) of the condenser. These enable two alternative modalities: bright-field (BF) (left), where the illumination in the angular range $\left[0, \bar{\theta}_{\mathrm{i}}^{\mathrm{BF}}\right]$ with $n_{1} \sin \bar{\theta}_{\mathrm{i}}^{\mathrm{BF}} \equiv \overline{\mathrm{NA}}_{\mathrm{i}}^{\mathrm{BF}}=\mathrm{NA}_{\mathrm{obj}}$ is fully collected by the objective, and dark-field (DF) (right), where the illumination in the angular range $\left[\underline{\theta}_{\mathrm{i}}^{\mathrm{DF}}, \bar{\theta}_{\mathrm{i}}^{\mathrm{DF}}\right]$ with $n_{1} \sin \underline{\theta}_{\mathrm{i}}^{\mathrm{DF}} \equiv$ $\underline{N A}_{i}^{\mathrm{DF}}>\mathrm{NA}_{\text {obj }}$ is not collected by the objective. In this work, we denote the maximum or minimum value of a variable with a line above or below the symbol, respectively. When a quantity depends on the illumination modality, the superscript $l \in\{\mathrm{BF}, \mathrm{DF}\}$ will be used. In BF images, the transmitted light 
results in a bright background; NanOs redirect and block some of the illumination by scattering and absorption and appear therefore as dark diffraction-limited spots. Note that a fraction of the scattering (red wave fronts) is also collected by the objective, on top of the transmitted light. In DF, where scattering alone is collected, NanOs appear as bright spots on a dark background instead. Further details on the experimental setup and protocol are provided in section S.I of the Supporting Information (SI).

In its essence, our method consists in correlating a BF transmission image and a DF scattering image of a $\mathrm{NanO}$ to measure its optical cross sections. Specifically, the DF signal is proportional to the scattered power, which can be referenced to the illumination intensity given by the $\mathrm{BF}$ background to quantify the scattering cross-section. Scattering can be then subtracted from the extinction cross-section measured in BF to isolate the absorption contribution. This procedure, however, involves some subtleties: (i) only a fraction of the total scattered power is collected within the acceptance angle of the objective; (ii) the incident intensity depends on the angular range of illumination, so that the DF reference is different from the BF background; (iii) the magnitude and directionality of scattering depends on the angular range of illumination, so that the scattering contribution to the $\mathrm{BF}$ extinction is different from the DF signal. These differences must be carefully accounted for in order to achieve an accurate quantitation of the cross-section magnitude. To this end, in the following section, we develop a rigorous description of our microspectroscopy experiments where the effects above are formally introduced via a set of parameters that depend on the experimental geometry and on the directional properties of the scattering. A model of the scattering process formulated under rather broad assumptions enables us to derive analytical expressions for all the parameters.

Measurement of the Cross-Section Magnitude. We measure a signal $S$ by integrating the sample image formed by the objective over a certain detection region $A$. $S$ is divided by the exposure time of the frame, and the dark offset of the camera is subtracted. The size of $A$ in the sample plane is determined via the known magnification of the imaging path. The NanO signal $S_{\mathrm{NO}}^{l}$ is measured over $A_{\mathrm{NO}}$, which must contain the $\mathrm{NanO}$ and no other significant absorbers or scatterers. The local background, $S_{\mathrm{bg}}^{l}$ is measured over $A_{\mathrm{bg}}$, which must be a region close to $A_{\mathrm{NO}}$ not containing significant absorbers or scatterers. Thus, under BF and DF illumination, four signals corresponding to the square frames in Figure 1 can be detected, namely, $S_{\mathrm{NO}}^{\mathrm{BF}}$, the NanO transmission in BF, $S_{\mathrm{NO}}^{\mathrm{DF}}$, the NanO scattering in DF, $S_{\mathrm{bg}}^{\mathrm{BF}}$, the transmitted illumination in $\mathrm{BF}$, and $S_{\mathrm{bg}}^{\mathrm{DF}}$, the diffuse scattering background in DF. Each signal is proportional to the power emerging from $A$ via the optical efficiency $\epsilon$ of the detection path: $P_{\mathrm{NO}}^{l}=S_{\mathrm{NO}}^{l} / \epsilon$ and $P_{\mathrm{bg}}^{l}$ $=\left(A_{\mathrm{NO}} / A_{\mathrm{bg}}\right) S_{\mathrm{bg}}^{l} / \epsilon . P_{\mathrm{bg}}^{l}$ includes an area scaling to represent $P_{\mathrm{NO}}^{l}$ in the absence of the $\mathrm{NanO}$. We now want to manipulate the definitions

$$
\sigma_{\mathrm{abs}}^{\mathrm{BF}} \equiv P_{\mathrm{abs}}^{\mathrm{BF}} / I_{\mathrm{i}}^{\mathrm{BF}} \quad \text { and } \quad \sigma_{\mathrm{sca}}^{\mathrm{DF}} \equiv P_{\mathrm{sca}}^{\mathrm{DF}} / I_{\mathrm{i}}^{\mathrm{DF}}
$$

to express $\sigma$ solely in terms of the four detected signals. Note that the values of $\sigma$ in eq 1 depend on the BF or DF illumination modality. We emphasize that in general the cross sections of a NanO depend on the excitation, particularly its polarization. For instance, elongated particles have a much larger cross-section under illumination polarized along their major axis. As a consequence, the cross sections can be different when measured in BF or DF, which have different polarization contents. Specifically, DF illumination impinges on the sample with higher angles and therefore contains a stronger longitudinal polarization component.

In eq 1 , the incident intensities traversing the sample plane, $I_{\mathrm{i}}^{\mathrm{BF}}$ and $I_{\mathrm{i}}^{\mathrm{DF}}$, are taken as reference. The incident power, $P_{\mathrm{i}}^{l}$, is proportional to them via the detection area: $P_{\mathrm{i}}^{l}=A_{\mathrm{bg}} I_{\mathrm{i}}^{l}$. Due to the presence of the interface, the illumination power $P_{\mathrm{bg}}^{\mathrm{BF}}$ measured in $\mathrm{BF}$ is less than $P_{\mathrm{i}}^{\mathrm{BF}}$ but still proportional to it. Let us then introduce the proportionality factor $\tau^{\mathrm{BF}} \equiv P_{\mathrm{bg}}^{\mathrm{BF}} / P_{\mathrm{i}}^{\mathrm{BF}}$, which can be computed as the transmittance $T$ across the interface averaged over the axially symmetric angular range of illumination. Now, we assume that the condenser lens is an aplanatic optical system illuminated by a homogeneous intensity over its BFP. As demonstrated in section S.IV A of the SI, this implies a $\cos \left(\theta_{\mathrm{i}}\right)$ dependence of the illumination power on the sample per solid angle, so that

$$
\tau^{\mathrm{BF}}=\frac{\int_{\underline{\theta}_{\mathrm{i}}^{\mathrm{BF}}}^{\bar{\theta}_{\mathrm{BF}}^{\mathrm{BF}}}\left[T_{\mathrm{p}}\left(\theta_{\mathrm{i}}\right)+T_{\mathrm{s}}\left(\theta_{\mathrm{i}}\right)\right] \cos \theta_{\mathrm{i}} \sin \theta_{\mathrm{i}} \mathrm{d} \theta_{\mathrm{i}}}{2 \int_{\underline{\theta}_{\mathrm{i}}^{\mathrm{BF}}}^{\bar{\theta}_{\mathrm{BF}}^{\mathrm{BF}}} \cos \theta_{\mathrm{i}} \sin \theta_{\mathrm{i}} \mathrm{d} \theta_{\mathrm{i}}}
$$

where the subscripts $\mathrm{p}$ and $\mathrm{s}$ of $T$ indicate a polarization of the incident electric field parallel or perpendicular to the plane of incidence. Conversely, in DF the transmitted light is not detected, and the incident intensity, $I_{\mathrm{i}}^{\mathrm{DF}}$, cannot be measured in the same way as in BF. However, $I_{\mathrm{i}}^{\mathrm{DF}}$ is proportional to $I_{\mathrm{i}}^{\mathrm{BF}}$ provided the source power is kept fixed, and thus we introduce the proportionality factor $\xi \equiv I_{\mathrm{i}}^{\mathrm{BF}} / I_{\mathrm{i}}^{\mathrm{DF}}$. For a homogeneous BFP illumination, $I_{\mathrm{i}}^{l}$ is proportional to the illuminated BFP region $A_{\mathrm{BFP}}^{l}$ and

$$
\xi=\frac{A_{\mathrm{BFP}}^{\mathrm{BF}}}{A_{\mathrm{BFP}}^{\mathrm{DF}}}=\frac{\sin ^{2} \bar{\theta}_{\mathrm{i}}^{\mathrm{BF}}}{\sin ^{2} \bar{\theta}_{\mathrm{i}}^{\mathrm{DF}}-\sin ^{2} \underline{\theta}_{\mathrm{i}}^{\mathrm{DF}}}
$$

where in the last equality we used the fact that for an aplanatic lens the radius in the BFP is proportional to $\sin \theta_{\mathrm{i}}$. Summarizing, the denominators of eq 1 are

$$
I_{\mathrm{i}}^{\mathrm{BF}}=P_{\mathrm{bg}}^{\mathrm{BF}} /\left(A_{\mathrm{bg}} \tau^{\mathrm{BF}}\right) \quad \text { and } \quad I_{\mathrm{i}}^{\mathrm{DF}}=I_{\mathrm{i}}^{\mathrm{BF}} / \xi
$$

Let us now turn our attention to the numerators and observe that only a portion, $P_{\mathrm{obj}}^{l}$, of the power scattered by the NanO over the whole $4 \pi$ solid angle is collected by the objective. When measuring the $\mathrm{NanO}$ scattering in $\mathrm{DF}$, one detects this portion on top of the background: $P_{\mathrm{NO}}^{\mathrm{DF}}=P_{\mathrm{obj}}^{\mathrm{BF}}+P_{\mathrm{bg}}^{\mathrm{DF}}$. Let us introduce the collected fraction $\eta^{l} \equiv P_{\mathrm{obj}}^{l} / P_{\mathrm{sca}}^{l}$, so that

$$
P_{\mathrm{sca}}^{\mathrm{DF}}=P_{\mathrm{obj}}^{\mathrm{DF}} / \eta^{\mathrm{DF}}=\left(P_{\mathrm{NO}}^{\mathrm{DF}}-P_{\mathrm{bg}}^{\mathrm{DF}}\right) / \eta^{\mathrm{DF}}
$$

By contrast, in $\mathrm{BF}$ one detects the transmitted illumination attenuated by the $\mathrm{NanO}$, as well as the collected portion of NanO scattering, $P_{\mathrm{NO}}^{\mathrm{BF}}=P_{\mathrm{bg}}^{\mathrm{BF}}-P_{\mathrm{ext}}^{\mathrm{BF}}+P_{\mathrm{obj}}^{\mathrm{BF}}$. By decomposing $P_{\mathrm{ext}}^{\mathrm{BF}}$ $=P_{\mathrm{sca}}^{\mathrm{BF}}+P_{\mathrm{abs}}^{\mathrm{BF}}$ one finds

$$
P_{\mathrm{abs}}^{\mathrm{BF}}=P_{\mathrm{bg}}^{\mathrm{BF}}-P_{\mathrm{NO}}^{\mathrm{BF}}-\left(1-\eta^{\mathrm{BF}}\right) P_{\mathrm{sca}}^{\mathrm{BF}}
$$

Now, $P_{\text {sca }}^{\mathrm{BF}}$ cannot be measured directly like its DF counterpart $P_{\text {sca. }}^{\mathrm{DF}}$. Indeed, due to the dependence of scattering on the angular range of illumination, they are in general different. Let us then introduce their ratio $\zeta \equiv P_{\text {sca }}^{\mathrm{BF}} / P_{\mathrm{sca}}^{\mathrm{DF}}$. Eventually, by substituting the expressions 4,5 , and 6 into eq 1 , we find the cross sections 
Table 1. Parameters Used for Quantitative Cross-Section Measurement and Their Values in the Experiments of This Work ${ }^{a}$

\begin{tabular}{lllllll} 
name & definition & \multicolumn{1}{c}{ meaning } & $(1)$ & $(2)$ & $(3)$ & $(4)$ \\
$\tau^{\mathrm{BF}}$ & $P_{\mathrm{bg}}^{\mathrm{BF}} / P_{\mathrm{i}}^{\mathrm{BF}}$ & transmission of the interface in BF & 1.00 & 1.00 & 0.934 & 0.934 \\
$\xi$ & $I_{\mathrm{i}}^{\mathrm{BF}} / I_{\mathrm{i}}^{\mathrm{DF}}$ & BF-to-DF ratio of incident intensity & 2.11 & 3.92 & 3.92 & 2.53 \\
$\eta^{\mathrm{BF}}$ & $P_{\mathrm{obj}}^{\mathrm{BF}} / P_{\mathrm{sca}}^{\mathrm{BF}}$ & fraction of scattering collected in BF & 0.136 & $0.148^{b}$ & $0.148^{b}$ & \\
$\eta^{\mathrm{DF}}$ & $P_{\mathrm{obj}}^{\mathrm{DF}} / P_{\mathrm{sca}}^{\mathrm{DF}}$ & fraction of scattering collected in DF & 0.112 & $0.148^{b}$ & $0.148^{b}$ & 0.127 \\
$\zeta$ & $P_{\mathrm{bg}}^{\mathrm{BF}} / P_{\mathrm{i}}^{\mathrm{BF}}$ & BF-to-DF ratio of scattered power & 1.45 & 3.69 & 2.26 & 0.847
\end{tabular}

${ }^{a}$ (1) Gold spheres in oil; (2) gold rods in oil; (3) gold rods in air; (4) polystyrene spheres in air. ${ }^{b}$ The similarity of $\eta^{l}$ in the two environments is coincidental. With respect to oil, in air a compensation occurs between less scattering going toward the collection side and a smaller $\theta_{\text {obj}}$.

$$
\begin{aligned}
\sigma_{\mathrm{sca}}^{\mathrm{DF}} & =\tau^{\mathrm{BF}} \frac{\xi}{\eta^{\mathrm{DF}}} \frac{A_{\mathrm{bg}} S_{\mathrm{NO}}^{\mathrm{DF}}-A_{\mathrm{NO}} S_{\mathrm{bg}}^{\mathrm{DF}}}{S_{\mathrm{bg}}^{\mathrm{BF}}} \\
\sigma_{\mathrm{abs}}^{\mathrm{BF}} & =\tau^{\mathrm{BF}} \frac{A_{\mathrm{NO}} S_{\mathrm{bg}}^{\mathrm{BF}}-A_{\mathrm{bg}} S_{\mathrm{NO}}^{\mathrm{BF}}}{S_{\mathrm{bg}}^{\mathrm{BF}}}-\left(1-\eta^{\mathrm{BF}}\right) \frac{\zeta}{\xi} \sigma_{\mathrm{sca}}^{\mathrm{DF}}
\end{aligned}
$$

which are independent of the optical efficiency $\epsilon$. Note that the cross sections measured under $\mathrm{BF}$ and $\mathrm{DF}$ are related via $\sigma^{\mathrm{BF}}=$ $(\zeta / \xi) \sigma^{\mathrm{DF}}$, so that the second term in eq 7a is the portion of BF scattering not collected by the objective: this must be subtracted from the total extinction (first term) to isolate the absorption contribution.

In eqs 7 , four parameters appear, whose definition and meaning are summarized in Table 1 . The illumination parameters, $\tau^{\mathrm{BF}}$ and $\xi$, are determined by the geometry of the experiment alone and are given by eq 2 and eq 3 respectively. By contrast, the scattering parameters, $\eta^{l}$ and $\zeta$, depend also on the properties of the $\mathrm{NanO}$ and its environment. In the following section, we show how to compute them.

Scattering Parameters of the Nano-object. Consider the power $P_{\text {det }}^{l}$ scattered within the polar angle range of detection $\left[\underline{\theta}_{\mathrm{d}}, \bar{\theta}_{\mathrm{d}}\right]$, which is given by the integral

$$
P_{\mathrm{det}}^{l}\left(\underline{\theta}_{\mathrm{d}}, \bar{\theta}_{\mathrm{d}}\right) \equiv \int_{\underline{\theta}_{\mathrm{d}}}^{\bar{\theta}_{\mathrm{d}}} \int_{0}^{2 \pi} \mathcal{P}_{\mathrm{NO}}^{l}(\theta, \varphi) \sin \theta \mathrm{d} \theta \mathrm{d} \varphi
$$

of the angular distribution $\mathcal{P}_{\text {NO }}^{l}$ of the power scattered by the $\mathrm{NanO}$ to the far field. The scattering parameters can be expressed in terms of $P_{\mathrm{det}}^{l}$ as

$$
\eta^{l}=\frac{P_{\mathrm{det}}^{l}\left(\theta_{\mathrm{obj}}, \pi\right)}{P_{\mathrm{det}}^{l}(0, \pi)} \quad \text { and } \quad \zeta=\frac{P_{\mathrm{det}}^{\mathrm{BF}}(0, \pi)}{P_{\mathrm{det}}^{\mathrm{DF}}(0, \pi)}
$$

Quite generally $\mathcal{P}_{\text {NO }}^{l}$, and hence the scattering parameters, can be computed with numerical techniques, as we will show in a future work. However, it is convenient to have approximate analytical expressions of $\eta^{l}$ and $\zeta$ for specific cases so that our quantitative method can be largely automated.

Our analytical description is based on the following assumptions: (i) The illumination is incoherent and homogeneous over the BFP of the condenser. (ii) The condenser is aplanatic. (iii) The NanO polarization is treated in the electrostatic approximation, namely, only dipolar resonant modes are considered. This describes well small NanOs with respect to the wavelength in the medium $\lambda / n$. (iv) Multiple scattering is neglected, which is justified for weak scatterers or weakly reflecting interfaces. Following assumptions i and ii, the microscope illumination is represented as an incoherent superposition of plane waves, $\mathbf{E}_{\text {exc }}$ incident from the directions $\left(\theta_{\mathrm{i}}, \varphi_{\mathrm{i}}\right)$ within the illumination cone, see section S.IV A of the SI for the mathematical details. According to assumption iii, each plane wave excites an oscillating electric dipole $\mathbf{p}=$ $\boldsymbol{\alpha} \mathrm{E}_{\text {exc }}\left(\theta_{\mathrm{i}}, \varphi_{\mathrm{i}}\right)$ at the NanO position, where $\boldsymbol{\alpha}$ is the polarizability tensor of the NanO. The angular distribution $\mathcal{P}$ of the power radiated by a dipole of arbitrary orientation in the vicinity of a dielectric interface (here given by the substrate surface) has been derived in a series of papers by Lukosz and Kunz. ${ }^{20-23}$ For an incoherent light source, $\mathbf{E}_{\text {exc }}$ waves with different $\left(\theta_{\mathrm{i}}, \varphi_{\mathrm{i}}\right)$ have a random relative phase, and so do the dipoles they excite, so that $\mathcal{P}_{\mathrm{NO}}^{l}$ is the integral of the scattered power of the dipole over the angular range of illumination

$$
\begin{aligned}
\mathcal{P}_{\mathrm{NO}}^{l}(\theta, \varphi) \propto & \int_{\underline{\theta}_{\mathrm{i}}^{l}}^{\bar{\theta}_{\mathrm{i}}^{l}} \int_{0}^{2 \pi} p^{2}\left(\theta_{\mathrm{i}}, \varphi_{\mathrm{i}}\right) \mathcal{P}\left(\theta_{\mathrm{i}}, \varphi_{\mathrm{i}}, \theta, \varphi\right) \\
& \sin \theta_{\mathrm{i}} \mathrm{d} \theta_{\mathrm{i}} \mathrm{d} \varphi_{\mathrm{i}}
\end{aligned}
$$

Note that not only the amplitude $p$ of the dipole but also its orientation depends on $\left(\theta_{\mathrm{i}}, \varphi_{\mathrm{i}}\right)$, which in turn influences $\mathcal{P}$. Equation 10 is then substituted in eq 8 in order to compute the scattering parameters via eq 9 for a given $\boldsymbol{\alpha}$. The mathematical treatment we just outlined shares several features with an analytical model of a scattering microscopy experiment that has recently been developed to simulate iSCAT measurements. ${ }^{16}$

In section S.V of the SI, we discuss in detail the analytical description outlined above and calculate the scattering parameters for diagonal forms of $\boldsymbol{\alpha}$ corresponding to simple geometries of the resonant modes: linear in the substrate plane, for example, $\left(\alpha_{x x}, \alpha_{y y}, \alpha_{z z}\right)$ in the direction $(1,0,0)$ or $(0,1,0)$, or normal to the substrate plane, $(0,0,1)$; planar isotropic in the substrate plane, $(1,1,0)$; and spherical, $(1,1,1)$. In a homogeneous medium $\left(n_{1}=n_{2}\right)$, closed-form solutions are obtained, whereas simple integrals are found in the more general case of $\mathrm{NanOs}$ on a substrate. We implemented these expressions in a MATLAB script, which computes $\tau^{\mathrm{BF}}, \xi, \zeta$, and $\eta^{l}$ given the geometry of the experiment and the form of $\boldsymbol{\alpha}$. This software has been used to investigate the dependence of the scattering parameters on $n_{2}$ for a $\mathrm{NanO}$ placed on a glass substrate $\left(n_{1}=1.52\right)$, see Figure 2 . Panel a shows that $\eta^{l}$, the fraction of scattering collected by the objective with $\theta_{\text {obj }}=$ $118^{\circ}$, is increasing with $n_{2}$ from slightly more than $10 \%$ for a $\mathrm{NanO}$ in air $\left(n_{2}=1.00\right)$, as most scattering goes toward the denser medium, to over $40 \%$ in index-matching oil $\left(n_{2}=1.52\right)$. Only for a spherical $\boldsymbol{\alpha}$ we have $\eta^{\mathrm{BF}} \neq \eta^{\mathrm{DF}}$, since the orientation of $\mathbf{p}$ depends on $\theta_{\mathrm{i}}$, and hence $\mathcal{P}_{\mathrm{NO}}$ depends on the illumination range. $\eta^{l}\left(n_{2}\right)$ has a point of inflection at $\mathrm{NA}_{\mathrm{obj}}=$ $n_{1}$, corresponding to $\theta_{\mathrm{obj}}=\pi-\arcsin \left(n_{1} / n_{2}\right)$, which is the critical angle of transmission from medium 2 to 1 .

The BF-to-DF scattering ratio $\zeta$, shown in Figure $2 \mathrm{~b}$, is ruled by the relative amplitude of the components of $\mathbf{E}_{\mathrm{exc}}$ in medium 2. In fact, the condenser partially transforms the $x$ polarization in the BFP into $y$ and $z$ components (see section S.III of the $\mathrm{SI}$ ), and this effect increases with $\theta_{\mathrm{i}}$, resulting in different 


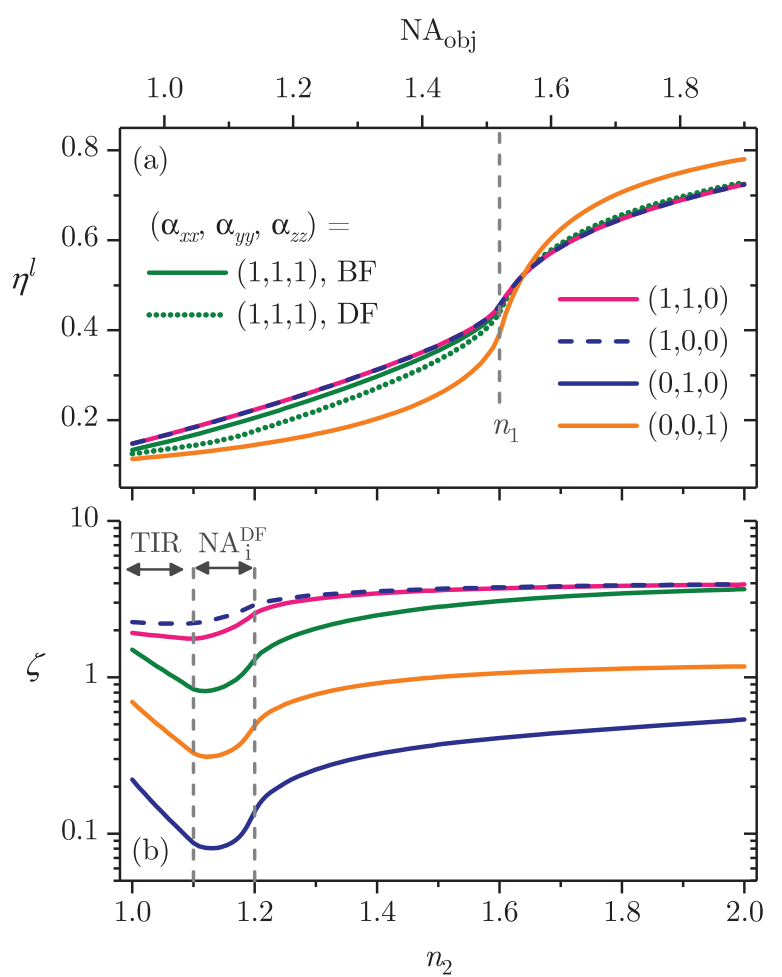

Figure 2. Scattering parameters (a) $\eta^{l}$ and (b) $\zeta$ against $n_{2}$ for several forms of the polarizability $\boldsymbol{\alpha}$ of the resonant mode. As illustrated in Figure 1, the $\mathrm{NanO}$ is deposited on a glass substrate $\left(n_{1}=1.52\right)$ and immersed in medium 2. The illumination comes from medium 1 and is polarized along $x$ in the BFP of the condenser. The illumination ranges are $\mathrm{NA}_{\mathrm{i}}^{\mathrm{BF}} \in[0,0.95]$ and $\mathrm{NA}_{\mathrm{i}}^{\mathrm{DF}} \in[1.1,1.2]$. The illumination undergoes complete TIR for $n_{2}<\underline{N A}_{i}^{\mathrm{DF}}$ and no TIR for $n_{2}>\overline{\mathrm{NA}}_{\mathrm{i}}^{\mathrm{DF}}$. The collection angle, $\theta_{\mathrm{obj}}=108^{\circ}$ has been kept fixed, resulting in a variable $\mathrm{NA}_{\mathrm{obj}} \equiv n_{2} \sin \theta_{\mathrm{obj}}$.

scattering efficiencies of $\mathrm{BF}$ and DF illumination. Indeed, the smallest values of $\zeta$, indicating a large DF scattering efficiency, are observed for dipoles oriented along $y$ and $z$. Moreover, the largest amplitude of $\mathbf{E}_{\text {exc }}$ in medium 2 occurs for close-tocritical total internal reflectance (TIR) illumination in DF, so that a minimum of $\zeta\left(n_{2}\right)$ occurs for $\mathrm{NA}_{\mathrm{i}}^{\mathrm{DF}}<n_{2}<\overline{\mathrm{NA}}_{\mathrm{i}}^{\mathrm{DF}}$, corresponding to a DF illumination range containing the critical angle of transmission from medium 1 to 2 . Overall, we find that $\zeta$ is more sensitive to $\boldsymbol{\alpha}$ than $\eta^{l}$, spanning more than 1 order of magnitude for some values of $n_{2}$.

\section{EXPERIMENTAL RESULTS}

As suggested by Figure 1, the detected signals can be decomposed into their chromatic components to measure cross-section spectra. We report here experimental results obtained via single $\mathrm{NanO}$ microspectroscopy, where a fine spectral resolution is provided by an imaging spectrometer and via high-throughput wide-field imaging of hundreds of NanOs, where a coarse spectral resolution is provided by optical filters.

Single-Particle Microspectroscopy. We used the microspectroscopy setup and measurement protocol described in section S.I of the SI to acquire the four signals required to measure the cross sections via eqs 7 . We present below results on nominally spherical gold particles (henceforth "spheres") and gold rods.

Gold Spheres. Nominally spherical gold particles (BBI solutions, EM.GC60) of diameter $D=60 \mathrm{~nm}$ were investigated. Figure 3 a shows a representative TEM micrograph of the batch we characterized optically and displays deviations from sphericity, variability in size, and crystal defects.

The colloid was drop-cast onto a glass coverslip $\left(n_{1}=1.52\right)$ and covered by index-matching silicone oil $\left(n_{2}=1.52\right)$ to create a homogeneous optical environment. Since the colloid was stabilized by citrate, no residual capping agent is expected after drying. We used the illumination ranges $\mathrm{NA}_{\mathrm{i}}^{\mathrm{BF}} \in[0,0.95]$ and $\mathrm{NA}_{\mathrm{i}}^{\mathrm{DF}} \in[1.10,1.28]$, yielding the parameter values reported in Table 1. $\sigma_{\mathrm{sca}}(\lambda)$ and $\sigma_{\mathrm{abs}}(\lambda)$ were determined using eqs 7 for 5 individual spheres under unpolarized illumination.

The single-particle spectra (solid lines in Figure 3b,c) exhibit a broad LSPR centered at $\lambda_{\mathrm{LSPR}} \simeq 555 \mathrm{~nm}$, in good agreement
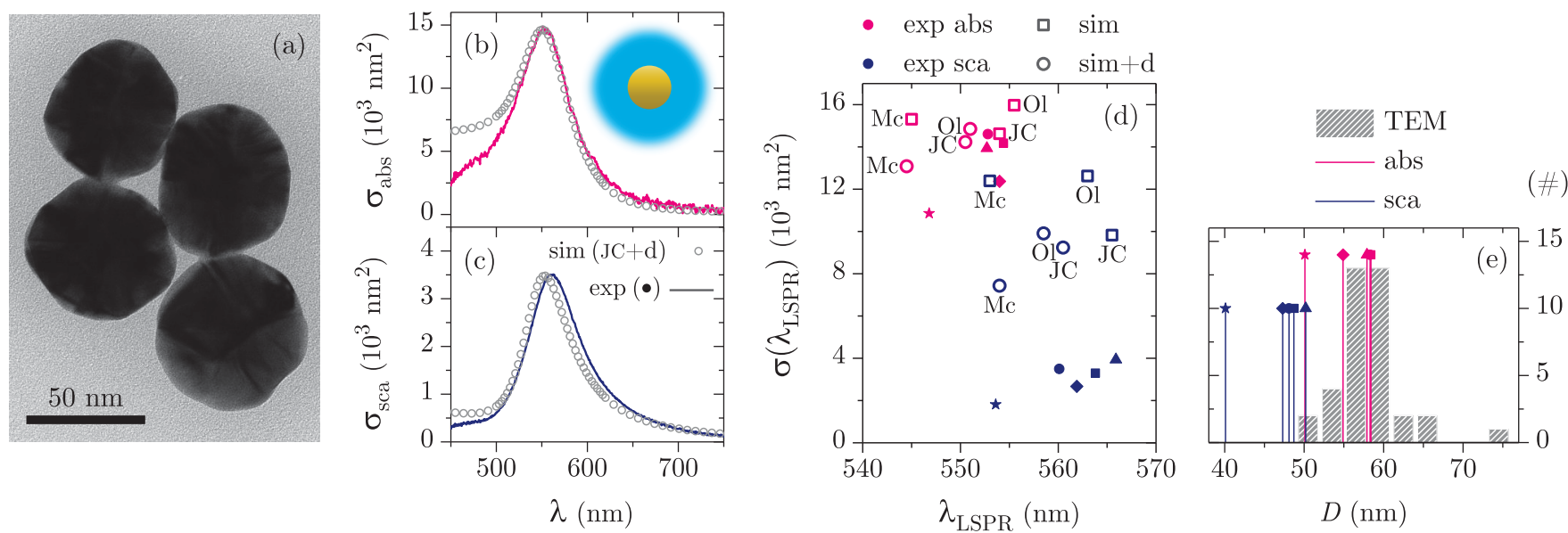

Figure 3. (a) Representative TEM micrograph of gold spheres of the measured batch. (b) Absorption and (c) scattering cross-section spectra of a single sphere (identified by the symbol $\bullet$ in panels $\mathrm{d}$ and e) in a homogeneous $n=1.52$ optical environment. The experimental data (solid lines) are fitted by numerical simulations $(\mathrm{O})$ using the sphere diameter $D$ as a free parameter. (d) LSPR peak position $\lambda_{\text {LSPR }}$ and $\sigma$ for the 5 measured spheres, identified by different full symbols. The hollow symbols are corresponding simulations for a sphere of diameter $D=58 \mathrm{~nm}$ and $\varepsilon(\lambda)$ after $\mathrm{JC},{ }^{24} \mathrm{Mc}^{25}$ and $\mathrm{Ol},{ }^{26}$ and the same with damping added $(+\mathrm{d})$ as described in the text. (e) Number distribution of $D$ measured with TEM over 37 spheres. The vertical lines are estimates of $D$ obtained by fitting the spectra in panels b and $\mathrm{c}$ with $\varepsilon(\lambda)$ after JC+d; different symbols identify the same individual spheres as in panel $\mathrm{d}$. 
with the vast literature on the subject. $\sigma_{\mathrm{abs}}(\lambda)$ is visibly noisier than $\sigma_{\text {sca }}(\lambda)$. For these absorption-dominated NanOs $\sigma_{\text {abs }} / A \simeq$ $1-P_{\mathrm{NO}}^{\mathrm{BF}} / P_{\mathrm{bg}}^{\mathrm{BF}}$, that is, a spatial modulation of the transmission reaching a maximum of about $1 \%$ at the LSPR. The observed noise in $\sigma_{\text {abs }}$ of about $500 \mathrm{~nm}^{2}$ RMS corresponds to a spatial modulation in the $10^{-4}$ range and is attributed to slow drifts of the instrumentation over the time-scale of the measurement of a few minutes, whereas the expected shot-noise for the measurements shown is slightly below $100 \mathrm{~nm}^{2}$. Note that the noise in $\sigma_{\text {ext }}$ can be reduced to below $1 \mathrm{~nm}^{2}$ by suited referencing techniques, ${ }^{27}$ showing that the noise of the $\sigma_{\mathrm{abs}}$ measurements presented here is not a fundamental limitation. The LSPR wavelength $\lambda_{\text {LSPR }}$ and the corresponding $\sigma\left(\lambda_{\text {LSPR }}\right)$ is plotted in Figure $3 \mathrm{~d}$ for the measured spectra (full symbols) in order to assess the effect of sample dispersity. We observe variations of about $10 \%$ in $\sigma_{\mathrm{abs}}, 20 \%$ in $\sigma_{\mathrm{sca}}$ and $10 \mathrm{~nm}$ in $\lambda_{\text {LSPR. }}$.

We can compare these results to numerical simulations performed with the model described in section S.II of the SI, taking into account the details of the measurement method. Note that $\sigma$ under microscope illumination is in general larger than $\sigma$ computed under plane-wave illumination, and in particular by a factor 1.12 and 1.63 for the BF and DF ranges considered, according to eq S24 of the SI. This originates from the definition of the cross sections in eqs 7 using the intensity traversing the sample plane, which is lower than the incident plane wave intensity by a factor $\cos \theta_{\mathrm{i}}$. Intuitively speaking, the larger cross-section observed corresponds to a longer shadow cast by the $\mathrm{NanO}$ onto the sample plane for oblique illumination, so we call this the long shadow effect. In a recent work, ${ }^{28}$ we verified experimentally on absorption-dominated gold spheres the dependence of $\sigma_{\text {ext }}$ on the angular range of illumination predicted by the long shadow argument.

To include the optical properties of gold in the model, we used several reported experimental permittivity, $\varepsilon(\lambda)$, data. ${ }^{24-26}$ We modified these data sets as described in section S.VII of the SI in order to best represent the material properties of each NanO. Briefly, we fitted the experimental $\varepsilon(\lambda)$ with a theoretical model ${ }^{29}$ and then increased the damping of the free electron term to account for the additional carrier scattering mechanisms introduced by crystalline defects and by the NanO surface, which are expected to be less relevant in the data measured by ellipsometry on thin films ${ }^{24,25}$ or single-crystalline bulk samples. ${ }^{26}$ The damping is chosen for each $\mathrm{NanO}$ to match measured and simulated line width of the LSPR. This approach and the resulting magnitude of the damping are consistent with previous experimental and theoretical works. ${ }^{29-32}$

The hollow symbols in Figure $3 \mathrm{~d}$ are simulations for a sphere of diameter $D=58 \mathrm{~nm}$ (TEM average, see Figure 3e) using the various $\varepsilon(\lambda)$ data sets, with and without added damping. Both amplitude and position of the simulated LSPR are close to the measurements for absorption, whereas $\sigma_{\mathrm{sca}}$ is smaller by a factor 3 with respect to simulations, which is larger than the variability between measurements of different spheres and between simulations with different $\varepsilon(\lambda)$. We will examine the possible reasons for this discrepancy in the Discussion and Conclusions section below. The good agreement observed in the peak position rules out ellipticity of the particles above a few percent in the sample plane. In fact, an increase of the aspect ratio (AR) above 1 is known $^{33}$ to entail $\sim 1 \mathrm{~nm} /$ 0.01(AR) linear red shift of the LSPR.

$D$ can be used as a free parameter in the simulations and adjusted to match the experimental $\sigma\left(\lambda_{\mathrm{LSPR}}\right)$. We call this fitting procedure optical sizing, whereby geometrical parameters of an individual $\mathrm{NanO}$ are determined by means of an optical measurement, and we propose it both for validation and as application of our quantitative method. Optical sizing of quasispherical particles has been reported ${ }^{30}$ comparing SMS measurements of $\sigma_{\text {ext }}(\lambda)$ to the theoretical spectrum of an ellipsoid in the electrostatic approximation under plane-wave illumination. For sizing, we used $\varepsilon(\lambda)$ after Johnson and Christy $^{24}$ with additional damping, because this data set reproduces best the observed $\lambda_{\text {LSPR }}$ see Figure S9 of the SI. $D$ determined for each sphere is compared in Figure $3 e$ to the size histogram resulting from the TEM characterization of the batch we investigated. The values obtained from $\sigma_{\text {abs }}\left(\lambda_{\text {LSPR }}\right)$ are consistent with the TEM distribution, whereas the values based on $\sigma_{\text {sca }}\left(\lambda_{\text {LSPR }}\right)$ are approximately $20 \%$ smaller, consistent with the deviations observed in Figure $3 \mathrm{~d}$.

The simulated $\sigma_{\mathrm{abs}}(\lambda)$ in Figure $3 \mathrm{~b}$ shows a good match to the experimental $\sigma_{\mathrm{abs}}(\lambda)$ in the LSPR region. The deviation for $\lambda<500 \mathrm{~nm}$ could be related to the reduced illumination power in this range combined with stray light in the spectrometer. The simulated $\sigma_{\text {sca }}(\lambda)$ in Figure $3 \mathrm{c}$, on the other hand, exhibits a slightly blue-shifted and narrower LSPR than the measured spectrum. This is ascribed to lower retardance effects, since the simulated sphere is about $10 \mathrm{~nm}$ smaller than the measured one. Indeed, in Figure $3 \mathrm{~d}$, where $D=58 \mathrm{~nm}$ is used for simulations, there is no spectral mismatch.

Gold Rods. Gold rods (Nanopartz, A12-25-650-CTAB) of nominal $\lambda_{\mathrm{LSPR}}=650 \mathrm{~nm}$ in water are investigated. Their typical size and geometry is exemplified by the TEM micrograph in Figure $4 a$. In contrast to the spheres in Figure 3a, the highresolution close-up of Figure $4 \mathrm{~b}$ displays a regular atomic lattice with no crystalline defects. For numerical simulation purposes, gold rods were modeled with an octagonal transverse section, based on TEM tomography studies on similar samples. ${ }^{34}$ In order to reproduce the geometry we observed by TEM, we used as end-caps right pyramids with a basis angle of $53^{\circ}$, truncated at height $W / 3$, where $W$ is the rod width (i.e., the span of the octagon). The rods are wrapped in a bilayer of cetyl-trimethylammonium-bromide (CTAB), a surfactant used to control the anisotropic growth of the crystalline seeds and stabilize the colloid. We modeled this bilayer as a dielectric shell of refractive index ${ }^{35} 1.435$ and homogeneous thickness ${ }^{36}$ $3.2 \mathrm{~nm}$.

The colloid was drop-cast on a glass slide $\left(n_{1}=1.52\right)$ and measured in air $\left(n_{2}=1.00\right)$ first; then the sample was covered by index-matching oil $\left(n_{2}=1.52\right)$, and the same rods were measured again to appraise the consistency of our method in different dielectric environments and in particular our ability to account for the presence of an interface. $\sigma_{\text {sca }}^{\mathrm{DF}}(\lambda)$ and $\sigma_{\mathrm{abs}}^{\mathrm{BF}}(\lambda)$ were measured for 7 individual rods under illumination polarized along the long rod axis $\left( \pm 5^{\circ}\right)$ in the BFP of the condenser. We used the illumination ranges $\mathrm{NA}_{\mathrm{i}}^{\mathrm{BF}} \in[0,0.95]$ and $\mathrm{NA}_{\mathrm{i}}^{\mathrm{DF}} \in[1.1,1.2]$, yielding the parameter values reported in Table 1. Representative single-rod spectra are shown in Figure $4 c, d$, and are dominated by the longitudinal LSPR, which red shifts as the refractive index of the immersion medium increases, as widely reported in literature. ${ }^{33}$ The measured LSPR position and amplitude of all individual rods (full symbols in Figure 4e) exhibit significant sample dispersity.

Again, we compare the quantitative measurements LSPRs to numerical simulations (hollow symbols in Figure 4e) using a rod having the typical size deduced from TEM ( $\mathrm{AR}=2.4$ and $W=28 \mathrm{~nm}$, see Figure $4 \mathrm{~g}$ ) and the material properties given by 

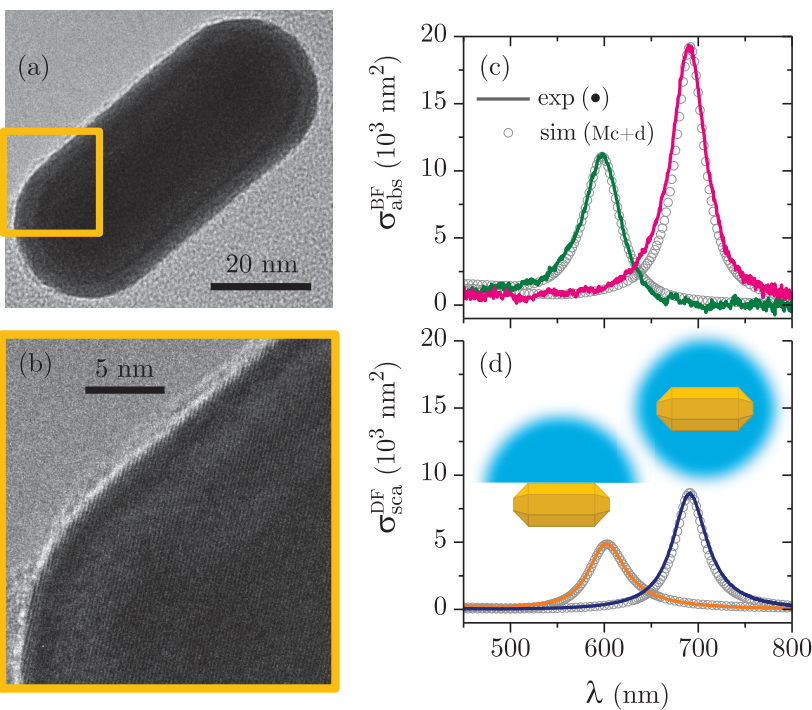

(e)

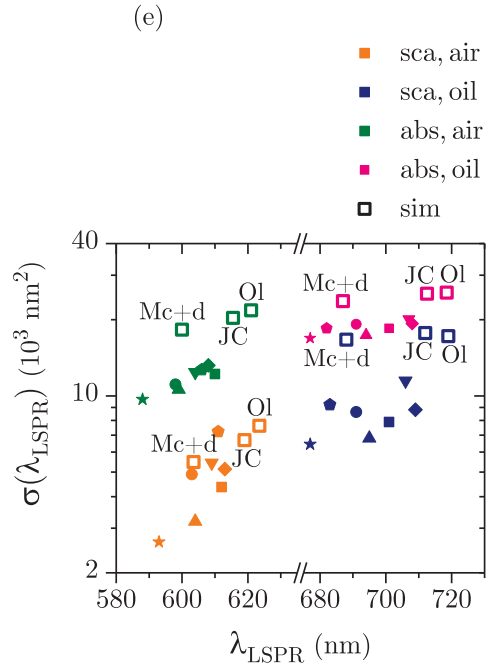

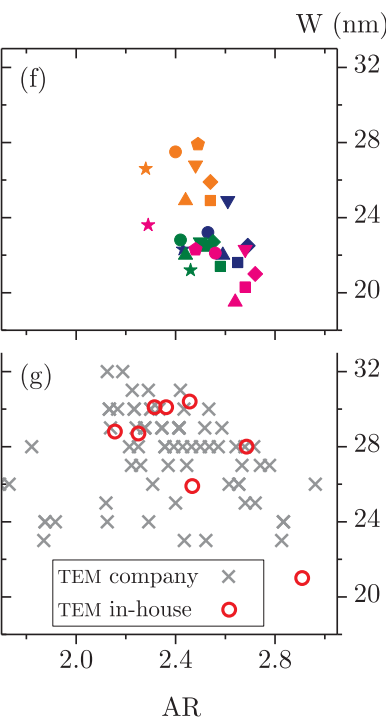

Figure 4. (a) Representative TEM micrograph of a gold rod of the measured batch. (b) Higher resolution micrograph of the framed region in panel a. (c) Absorption and (d) scattering cross-section spectra of a single rod (identified by the symbol $\bullet$ in the panels e and f) deposited on a glass substrate $\left(n_{1}=1.52\right)$ and immersed in air $\left(n_{2}=1.00\right)$ or index-matching oil $\left(n_{2}=n_{1}\right)$ (short and long wavelength peak, respectively). The illumination was polarized along the rod long axis in the BFP of the condenser. The experimental data (solid lines) are fitted by numerical simulations (hollow circles) using the rod aspect ratio, AR, and width, $W$, as free parameters. (e) LSPR peak position, $\lambda_{\text {LSPR }}$ and absolute amplitude, $\sigma$, for the 7 measured rods, identified by different full symbols. The hollow symbols are simulations for a rod of $A R=2.4$ and $W=28$ $\mathrm{nm}$, and $\varepsilon(\lambda)$ after $\mathrm{JC}^{24}$ or $\mathrm{Mc}^{25}$ with added damping $(\mathrm{Mc}+\mathrm{d})$, or $\mathrm{Ol}^{26}$ Color coding in panels $\mathrm{c}-\mathrm{f}$ refers to the legend above panel e. (f) AR and $W$ deduced from independent fits of the four spectra; the symbols identify the same individual rods as in panel e. (g) AR and $W$ measured from TEM micrographs of the measured batch. Crosses ( 80 rods, a few falling outside the plotted range) refer to images provided by the manufacturer, while circles ( 9 rods) refer to images taken in house.

the experimental $\varepsilon(\lambda)$ data sets already considered for the spheres. We reproduced the incoherent microscope illumination of experiments with a weighted average of simulations with the incidence direction of the exciting plane wave varying over the experimental illumination range. Our analytical description of the microscope illumination and the resulting averaging formulas are presented in section S.IV of the SI. Conversely, most previous works compare their experimental results to simulations under plane wave illumination with normal incidence to the substrate, thereby neglecting the long shadow effect and the polarization component normal to the substrate, which is significant for high NA illumination.

Similar to the spheres, in Figure $4 \mathrm{e}$ the measured $\sigma\left(\lambda_{\mathrm{LSPR}}\right)$ are smaller than the simulations beyond the variability due to the sample dispersity for the former and the choice of the permittivity for the latter. $\sigma_{\text {sca }}^{\mathrm{DF}}$ in air makes an exception, but some compensation with other effects due to our analytical model of scattering may have taken place. For instance, we neglect the finite distance (order of $10 \mathrm{~nm}$ ) between the excited dipoles and the interface: this is expected to lead to an underestimate of $\eta$, and hence an overestimate of $\sigma_{\text {sca }}$. By modifying, within a realistic range, the geometry (transverse section and cap shape of the rod, distance from the interface, thickness of $\mathrm{CTAB}$ layer) or the material specifications (permittivity of gold, refractive index of CTAB) it is possible to reach a good agreement for one cross-section, say $\sigma_{\mathrm{abs}}^{\mathrm{BF}}\left(\lambda_{\mathrm{LSPR}}\right)$, in air. However, a simultaneous agreement for all four cross sections could not be reached. This exemplifies how the surplus information provided by quantitative and correlative measurements brings about a more stringent appraisal of the relation between structural parameters and optical properties. Similar to our results on $\sigma_{\mathrm{sca}}$ a recent iSCAT study ${ }^{17}$ of these rods (same manufacturer and nominal geometry) reported a $20 \%$ lower scattering intensity than the numerical prediction, indicating possible shortcomings in the modeling of these systems, see the Discussion and Conclusions section.

For the rods, we performed a two-parameter optical sizing by adjusting $\mathrm{AR}$ and $W$ to fit both position and amplitude of the LSPR. We used $\varepsilon(\lambda)$ after McPeak et al. ${ }^{25}$ as it reproduces better the LSPR position for the typical TEM geometry. Before fitting (i.e., still based on the TEM geometry), the damping of $\varepsilon$ was adjusted to match the line width of each measured spectrum. In general, less additional damping is required for rods (see Figure S9 of the SI), although the surface contribution is expected to be similar; this suggests that the dominating contribution is rather due to crystal defects for spheres and chemical interface damping for rods. ${ }^{37} \sigma_{\text {sca }}^{\mathrm{DF}}(\lambda)$ and $\sigma_{\mathrm{abs}}^{\mathrm{BF}}(\lambda)$ in air and in oil have been fitted independently, thereby producing four estimates of the geometry of each measured rod, displayed in Figure 4f. The sizing results can be compared to the TEM characterization of the colloid in Figure 4g. The estimated AR, which is mostly determined by the LSPR position, is in good agreement, whereas $W$ is smaller, consistent with the lower measured cross-section of the LSPR observed in Figure 4e. A good fit is obtained across the whole spectrum, see Figure $4 \mathrm{~b}, \mathrm{c}$, although the simulated LSPR are slightly narrower since the sizes determined are smaller than the typical TEM bringing about less radiative damping.

Optical sizing of similar gold rods based on SMS measurements ${ }^{38}$ fitted the measured $\sigma_{\text {ext }}$ spectra with simulations of $\sigma_{\mathrm{abs}}$ using a rod of circular transverse section and hemispherical end-caps. The authors determined a diameter of $25.5 \mathrm{~nm}$ and a length of $50 \mathrm{~nm}$, in rough agreement with ensemble TEM characterization (diameter 
15-20 nm, length 40-60 nm, aspect ratio 2-4). Two connected studies $^{39,40}$ used SMS to measure $\sigma_{\text {ext }}$ spectra and electron microscopy to measure the geometry of the same $\mathrm{NanO}$ on a $40 \mathrm{~nm}$ thick silica TEM substrate. They studied the effect of the environment, comparing gold rods in air, either bare or encapsulated in a thick $(10-15 \mathrm{~nm})$ silica shell, ${ }^{39}$ and different particle geometries, measuring bare rods and bipyramids in air. ${ }^{40}$ The measured $\sigma_{\text {ext }}$ spectra were compared to $\sigma_{\text {abs }}$ spectra simulated with a model including the thin TEM

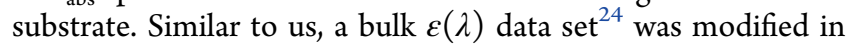
the model by adjusting the free electron damping to fit the average experimental line width of the LSPR. While an overall satisfactory agreement was found for the encapsulated rods, for all the particles in air, the LSPR systematically displayed a large (about $100 \mathrm{~nm}$ ) red shift with respect to simulations. To reproduce these results with simulations, the authors appeal to an effective refractive index $\tilde{n}$ of the air environment estimated to be $\tilde{n}=1.4$ for the rods and $\tilde{n}=1.1$ for the bipyramids. Such large values are attributed to the presence of surfactant residuals and of a thick (about $20 \mathrm{~nm}$ ) water layer enclosing the rods (but not the bipyramids). In contrast, we observe in Figure $4 \mathrm{e}$ that the LSPR shift of the same rod between air and oil is reproduced well using the nominal $n$ for simulations. We also note that in the numerical model of these works the lateral boundary conditions partially reflect the scattered radiation, and excitation is provided by a plane wave at normal incidence, whereas the SMS experiment used a tightly focused (0.75 NA) laser beam.

More generally, let us emphasize that it is often possible ${ }^{38-40}$ to reproduce numerically optical measurements by assuming geometric and material properties beyond the actual knowledge of the system and therefore determine these properties optically. However, for all but the simplest systems, the parameter space of structural properties is not well constrained by a single measurement. Addressing multiple observables for the same $\mathrm{NanO}$, such as $\sigma_{\mathrm{sca}}$ and $\sigma_{\mathrm{abs}}$, and correlating different environments, such as air and oil, as we did in this work, imposes much more stringent constraints and hence improves the reliability of the optically deduced structural properties.

High-Throughput Wide-Field Imaging. The quantitation formulas 7 do not refer specifically to dispersive microspectroscopy. Indeed the required signals can be acquired as well with conventional wide-field imaging. This permits simultaneous measurement of up to several hundred $\mathrm{NanOs}$ in one field of view, the density being limited by the interparticle spacing required to resolve and analyze individual NanOs. Image analysis was performed via the ImageJ plug-in Extinction Suite. ${ }^{41}$ This measurement and analysis technique has already been described elsewhere; ${ }^{42,43}$ for completeness, a short description of its rationale is reported in section S.I of the SI. An improved version of the technique ${ }^{27}$ showed shot noiselimited sensitivity down to $\sigma_{\text {ext }}=0.4 \mathrm{~nm}^{2}$. The previously published method ${ }^{42}$ analyzes BF and DF images to determine $\sigma_{\text {ext }}$ and $\sigma_{\text {sca }}$ using a single factor (also called $\eta$ ) to scale the measured $\sigma_{\text {sca }}$ which was deduced phenomenologically for spheres in homogeneous environment from the observed scaling of $\sigma_{\text {ext }}$ and $\sigma_{\text {sca }}$ with diameter. The quantitative method presented in this work addresses a more general situation, by introducing a set of parameters deduced exclusively from the experimental geometry and the symmetry of the resonant mode.

Polystyrene spheres (Polysciences, Polybead microspheres, cat. no. 00876) of nominal diameter $D=100 \mathrm{~nm}$ have been used to test the accuracy of our method when applied to dielectric NanOs. In contrast to most metal colloids, these have a very regular shape and homogeneous composition. The manufacturer size specification has been confirmed by a commercial dynamic light scattering (DLS) apparatus (Malvern, ZetaSizer Nano ZS), which reported Z-average sizes of $D_{z}=100.4$ and $96.5 \mathrm{~nm}$ in two successive runs. Furthermore, having a refractive index of $n_{\mathrm{PS}}=1.59$, their permittivity is nonresonant and dominated by electronic excitations localized on individual styrene rings, such that effects of surface scattering or nonlocality on the permittivity, relevant for the plasmonic particles, can be neglected. The colloid was drop-cast onto a glass coverslip $\left(n_{1}=1.52\right)$ and measured in air $\left(n_{2}=1.00\right)$. We used the illumination ranges $\mathrm{NA}_{\mathrm{i}}^{\mathrm{BF}} \in[0,0.95]$ and $\mathrm{NA}_{\mathrm{i}}^{\mathrm{DF}} \in[1.1,1.3]$, yielding the parameter values reported in Table 1 . $\xi$ was corrected to take into account the reduced transmission of the condenser at large NAs, see the point (M-ii) in the discussion below; the value according to eq 3 would be $\xi=1.88$. Figure 5a displays
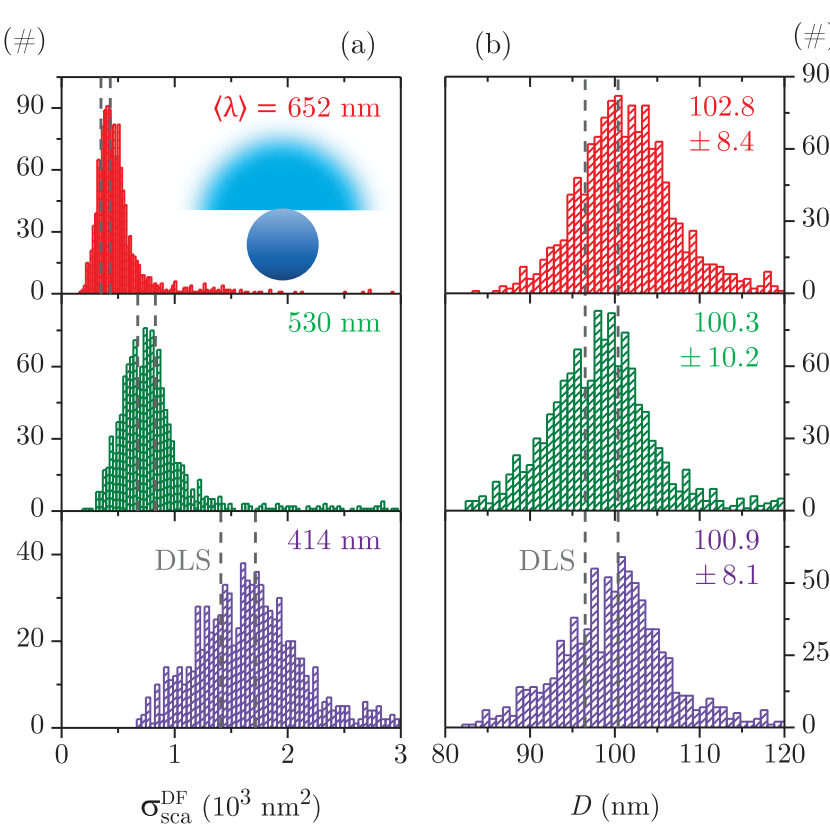

Figure 5. (a) Number distribution of the scattering cross-section of about 1000 individual polystyrene spheres deposited on a glass/air interface $\left(n_{1}=1.52, n_{2}=1.00\right)$. The three panels refer to different average excitation wavelengths, $\langle\lambda\rangle$. (b) Number distribution of the sphere diameter, $D$, deduced by comparison of data in panel a with numerical simulations of, $\sigma_{\mathrm{sca}}^{\mathrm{DF}}(D)$. The mean diameter and the standard deviation of each distribution are reported in the frames. The dashed lines indicate $D$ measured by DLS in panel $\mathrm{b}$, and the corresponding computed $\sigma_{\mathrm{sca}}^{\mathrm{DF}}(D)$ in panel a.

the $\sigma_{\text {sca }}^{\mathrm{DF}}$ distribution of around 1000 individual spheres measured at different excitation wavelengths over two fields of view. Note that $\sigma_{\text {sca }}^{\mathrm{DF}}$ is much smaller with respect to the metal NanOs studied above, in spite of these spheres being bigger, due to the absence of LSPRs. We ascribe the tails to higher values observed in the $\sigma_{\text {sca }}^{\mathrm{DF}}$ distribution to dielectric debris or particle aggregates. The decrease of $\sigma_{\text {sca }}$ with $\lambda$ is close to the expected ${ }^{1}$ scaling $\sigma_{\text {sca }} \propto \lambda^{-4}$ in the Rayleigh regime $D \ll \lambda$.

We performed numerical simulations of the cross sections for spheres of varying size, and fitted the results with the function $\sigma_{\mathrm{sca}}^{\mathrm{DF}}(D)=A D^{p}$. $A$ and $p$ were found to depend slightly 
on $\lambda$, approaching the Rayleigh dependence $p=6$ for large wavelengths. These relations were used to transform the distribution of $\sigma_{\mathrm{sca}}^{\mathrm{DF}}$ in Figure 5a into the distribution of $D$ in Figure $5 \mathrm{~b}$. The measured $\sigma_{\mathrm{sca}}^{\mathrm{DF}}$ is consistent with the simulations based on the DLS size (dashed lines in Figure 5a), within the width of the distribution, and consequently the estimated size is consistent with the DLS size (dashed lines in Figure 5b). The mean value of $D$ varies within a few percent between the three spectral channels.

A previously reported method ${ }^{44}$ for optical sizing of polystyrene spheres relies on the spectral position of the Mie resonances rather than the absolute scattering intensity and is therefore adequate only when applied to larger $(D \approx \lambda)$ spheres.

\section{DISCUSSION AND CONCLUSIONS}

In this work, we report an experimental procedure and analysis method for measuring the magnitude of $\sigma_{\mathrm{sca}}$ and $\sigma_{\mathrm{abs}}$ of a single $\mathrm{NanO}$ by combining BF and DF images acquired with a commercial optical microscope. We applied our approach in conjunction with two widely available experimental techniques: dispersive microspectroscopy, which provides fine spectral resolution, and spectrally filtered wide-field imaging with automated analysis, which offers a high-throughput and high-sensitivity characterization. We performed quantitative measurements on three technologically relevant model systems covering a wide range of features: metallic or dielectric material, spherical or elongated shape, homogeneous environment or presence of a substrate. Note that we deliberately do not address here metal NanOs smaller than a few tens of nanometers, which are harder to detect but whose crosssection is easier to quantify inasmuch as scattering is negligible. Indeed, other available techniques such as SMS and PHI, as well as our own extinction image analysis, ${ }^{27}$ already offer sensitive detection and accurate quantification for absorptiondominated NanOs. In this work, we focus instead on the more complicated scenario where $\sigma_{\text {sca }}$ and $\sigma_{\text {abs }}$ have comparable magnitude, and put forward an accurate way to quantify scattering and unravel its contribution to the extinction.

We compare our experimental results to numerical simulations, which include the dielectric substrate and implement a realistic description of the experimental illumination. Along with a remarkably good agreement between the measured and simulated cross-section magnitude in some cases (with relative differences below 10\%), we also observe in other cases relative differences up to a factor 3 . These differences can originate from systematic errors in measurements (M) and from approximations and missing details in simulations $(S)$; let us then list the main possible sources. In measurements, (M-i) the illumination NA ranges are defined by apertures in the BFP of the condenser, and the limits we reported come with an estimated error of \pm 0.02 . This is sizable for the DF range, which spans $0.1-0.2$ NA and affects $\xi$, and hence $\sigma_{\text {sca }}$ up to approximately $25 \%$, whereas only minor variations are expected in the other parameters. (M-ii) Our analytical description of the microscope illumination assumes that a homogeneous illumination over the BFP is focused by a perfectly aplanatic condenser. This yields an intensity over the sample plane independent of $\theta_{\mathrm{i}}$, see section S.IV A of the SI. However, according to characterization reported in section S.VI of the SI, in our instrument, the intensity reduces significantly for $\mathrm{NA}_{\mathrm{i}} \gtrsim 1.1$, mostly due to a lower transmission of the condenser. The main effect is a reduced DF illumination, which entails a larger $\xi$ and $\sigma_{\text {sca }}$ by up to $35 \%$ in the case of the polystyrene spheres. (M-iii) While the PSF of a NanO has extended tails, we only detect the scattering or extinction signal over a small region, $A_{\mathrm{NO}}$, of the sample image. This implies that a portion of the signal is not detected, resulting in an underestimate of the cross sections. In our experiments, we estimate to collect $80 \%$ to $90 \%$ of the total signal based on an Airy PSF model. For wide-field data, we are able to vary $A_{\mathrm{NO}}$ in the analysis to determine these factors as described in section S.I of the SI and use them to correct the measured $\sigma_{\text {sca. }}^{\mathrm{DF}}$. The error sources $\mathrm{M}$-i to M-iii can be mitigated by improving the experimental procedure and by characterizing the relevant instrument properties and using them to correct the parameters. In particular, we have corrected for Mii and M-iii in the analysis of the polystyrene spheres but not for the metal particles, which appear to be dominated by other larger systematics. (M-iv) We computed the scattering parameters $\eta^{l}$ and $\zeta$ used to analyze the measurements via a dipolar scattering model, which applies to $\mathrm{NanOs}$ within the electrostatic limit and described by simple forms of the polarizability. The resulting errors therefore depend on the $\mathrm{NanO}$ geometry and are in general expected to increase with the NanO size. In a follow-up work, we will show how to compute the scattering parameters through numerical simulations, to account for multipolar electric resonant modes, as well as for magnetic modes in high-refractive index dielectric NanOs. By overcoming the assumptions underlying the scattering treatment adopted in this work, the scope of the quantitative method can be extended to cover NanOs of arbitrary shape and material properties and size above the electrostatic limit.

A number of approximations that can lead to systematic errors are involved in simulations too. (S-i) Representing a $\mathrm{NanO}$ with simple geometric primitives means deviating from its real shape. This is particularly evident in the present examples for the gold spheres, which display rather irregular shapes (see Figure 3a) and whose response then could be influenced by plasmonic hot spots occurring at sharp features. (S-ii) The material description used might not represent well the optical response of the NanO. In particular, a local permittivity function could not be well-defined at the length scale of the investigated metal particles. ${ }^{45}$ For instance, we have increased the damping of the Drude part of the permittivity as discussed above: this is indeed a nonlocal effect well-known in the plasmonics field. (S-iii) Thin surface layers of dielectric materials could be present, such as water or organic residues from drop-casting. This would affect mostly the $\mathrm{NanO}$ in air, where this layer significantly changes the local susceptibility. In oil instead, water layers are not expected, and organic residues have a similar refractive index, so that the effect is expected to be small.

Let us summarize which sources of systematic errors could impact significantly $(\gtrsim 10 \%)$ the various systems we have investigated. For the gold spheres in oil, M-ii, S-i, and S-ii are expected to be relevant. For the gold rods, M-i, S-i, S-ii, and additionally S-iii in air only, are relevant. For the polystyrene spheres in air, M-ii, M-iv (specifically the finite distance to the substrate), and S-iii are relevant. On top of that, M-iii is relevant for all the systems.

Overall, the method we propose offers several advantages with respect to the single-NanO techniques we reviewed in the introduction; below we recapitulate the main ones. (i) Ease of use. Our method relies on a setup much less costly and 
complex that those used, for example, by SMS or PHI. Moreover, the analysis to retrieve the cross-section magnitude is almost fully automated at the user's end, and thus requires little specific skills. (ii) Accurate quantitation. Our method can measure the magnitude of both $\sigma_{\mathrm{sca}}$ and $\sigma_{\mathrm{abs}}$ of a broad range of $\mathrm{NanO}$ systems, also taking into account the presence of the substrate as an optical interface in the vicinity of the particle. In contrast, techniques such as conventional SMS and PHI are limited to absorption-dominated NanOs; additionally, their accuracy is affected by systematic errors related to several underlying assumptions (e.g., on the exciting electromagnetic field and on the thermal properties of the NanO environment), which are seldom evaluated. Quantitative measurements of $\sigma_{\text {sca }}$ on the other hand, are not yet well-established, with just two reports ${ }^{11,13}$ to date. (iii) High throughput. Our method, applied in conjunction with our automated image analysis technique, is capable of measuring many hundreds of NanOs at once, as shown above for the polystyrene spheres. It can thereby offer a robust statistical characterization of large ensembles (such as nanoparticle colloids) at the single object level. In contrast, techniques that form an image by rasterscanning a laser spot or a pinhole image on the sample measure NanOs sequentially and are therefore slower.

As an application of quantitative cross-section measurements, in this work, we demonstrate optical sizing, whereby some structural parameter of the measured $\mathrm{NanO}$ can be determined via a systematic comparison with accurate numerical simulations of the experiment. Essentially, this procedure permits achievement of nanometer-scale spatial resolution with optical microscopy. To date in literature, optical characterization of $\mathrm{NanO}$ geometry was mostly based on the shape of the optical spectra or its polarization properties, for example, in the case of metal rods, whose longitudinal $\lambda_{\text {LSPR }}$ increases with the AR. However, additional information on the cross-section magnitude can bring about a more precise and reliable determination of the structural parameters in many cases of practical interest. For instance, for small dielectric particles and small metal spheres, size variations do not alter significantly the shape of the optical spectra but only the cross-section magnitude. Moreover, $\sigma_{\text {sca }} \propto$ $D^{6}$ and $\sigma_{\text {abs }} \propto D^{3}$, which designates $\sigma_{\text {sca }}$ as a candidate for highprecision optical sizing.

We emphasize that quantitative cross sections measurements at the single-NanOs level and comparison with simulations accurately modeling the measurement conditions are a field yet in its infancy. Indeed, previous reports of optical sizing based on the cross-section magnitude ${ }^{30,38-40}$ often highlight significant inconsistencies with TEM data, or reach a good agreement by using model parameters that are not verified independently. Furthermore, these works relied on SMS measurements and are therefore limited to absorptiondominated NanOs. We believe that refining the presented quantitative method by reducing the errors listed above can offer a novel and important characterization tool for single NanOs and enable novel physical insights into the validity of theoretical models on the one hand and the material properties on the other hand.

As an outlook, quantitative cross-section measurements provide an important tool to assess and tailor the optical properties of NanOs for applications, specifically those demanding an efficient radiation-NanO coupling, such as photothermal and photovoltaic devices. They permit accurate comparison of measurements performed with different set-ups and allow testing more thoroughly the predictions of theoretical and numerical models. Overall, they foster a deeper fundamental understanding of the nanoplasmonics phenomenology and ease the translation to technology, for example, in nanoparticle fabrication. Thanks to its operational simplicity and low cost, the method presented here has the potential to be widely adopted, for instance, by industries and research groups synthesizing metal colloids or fabricating lithographic NanOs. In particular, high-throughput optical sizing via automated image analysis can provide an efficient and rapid all-optical structural characterization of a sample, reducing the need for more costly and time-consuming electron microscopy.

\section{ASSOCIATED CONTENT}

\section{Supporting Information}

The Supporting Information is available free of charge on the ACS Publications website at DOI: 10.1021/acsphotonics.9b00727.

Detailed description of the experiment, analysis, and simulation methods, specifically including (i) experimental setup and measurement protocol, (ii) model used for numerical simulations, (iii) derivation of the electric field above and below a dielectric interface, (iv) analytical description of the microscope illumination, ( $\mathrm{v}$ ) calculation of the scattering parameters, (vi) characterization of the experimental illumination, and (vii) modification of the permittivity of gold with additional damping and description of open data available (PDF)

\section{AUTHOR INFORMATION}

\section{Corresponding Author}

*E-mail: attilio.zilli@polimi.it.

ORCID 우

Attilio Zilli: 0000-0003-1845-6850

Wolfgang Langbein: 0000-0001-9786-1023

Paola Borri: 0000-0002-7873-3314

\section{Present Address}

${ }^{\S}$ A.Z.: Department of Physics, Politecnico di Milano, Piazza Leonardo da Vinci 32, 20133 Milano, Italy.

\section{Notes}

The authors declare no competing financial interest.

The raw and analyzed data discussed in this work, the MATLAB codes used for the analysis, and the comsol models used for numerical simulations are freely available in the Cardiff University Research Portal. ${ }^{46}$

\section{ACKNOWLEDGMENTS}

We thank our colleagues Lukas Payne for discussion and assistance with using Extinction Suite, Francesco Masia for calculating the modified permittivity data sets, and Thomas Davies for performing electron microscopy. We acknowledge funding from the European Commission, Research Executive Agency (Marie Curie Action 607842 FINON ITN-2013), the United Kingdom Engineering and Physical Sciences Research Council (Grants EP/I005072/1, EP/M028313/1, and EP/ R51150X/1), and the Welsh Government Life Sciences Bridging Fund (Grant LSBF/R6-005). P.B. acknowledges the Royal Society for her Wolfson research merit award (Grant WM140077). 


\section{REFERENCES}

(1) Bohren, C. F.; Huffman, D. R. Absorption and Scattering of Light by Small Particles; Wiley-VCH Verlag, 1998.

(2) Ringe, E.; Sharma, B.; Henry, A.-I.; Marks, L. D.; Van Duyne, R. P. Single nanoparticle plasmonics. Phys. Chem. Chem. Phys. 2013, 15, 4110.

(3) Dreaden, E. C.; Alkilany, A. M.; Huang, X.; Murphy, C. J.; ElSayed, M. A. The golden age: Gold nanoparticles for biomedicine. Chem. Soc. Rev. 2012, 41, 2740-2779.

(4) Mayer, K. M.; Hafner, J. H. Localized Surface Plasmon Resonance Sensors. Chem. Rev. 2011, 111, 3828-3857.

(5) Atwater, H. A.; Polman, A. Plasmonics for improved photovoltaic devices. Nat. Mater. 2010, 9, 205-213.

(6) Siedentopf, H.; Zsigmondy, R. A. Uber Sichtbarmachung und Größenbestimmung ultramikoskopischer Teilchen, mit besonderer Anwendung auf Goldrubingläser. Ann. Phys. 1902, 315, 1-39.

(7) Crut, A.; Maioli, P.; Del Fatti, N.; Vallée, F. Optical absorption and scattering spectroscopies of single nano-objects. Chem. Soc. Rev. 2014, 43, 3921-3956.

(8) Olson, J.; Dominguez-Medina, S.; Hoggard, A.; Wang, L.-Y.; Chang, W.-S.; Link, S. Optical characterization of single plasmonic nanoparticles. Chem. Soc. Rev. 2015, 44, 40-57.

(9) Arbouet, A.; Christofilos, D.; Del Fatti, N.; Vallée, F.; Huntzinger, J. R.; Arnaud, L.; Billaud, P.; Broyer, M. Direct Measurement of the Single-Metal-Cluster Optical Absorption. Phys. Rev. Lett. 2004, 93, 127401.

(10) Berciaud, S.; Cognet, L.; Blab, G. A.; Lounis, B. Photothermal Heterodyne Imaging of Individual Nonfluorescent Nanoclusters and Nanocrystals. Phys. Rev. Lett. 2004, 93, 257402-257405.

(11) Husnik, M.; Linden, S.; Diehl, R.; Niegemann, J.; Busch, K.; Wegener, M. Quantitative Experimental Determination of Scattering and Absorption Cross-Section Spectra of Individual Optical Metallic Nanoantennas. Phys. Rev. Lett. 2012, 109, 233902.

(12) The quantitative analysis by Husnik et al. ${ }^{11}$ assumes a $\mathrm{NanO}$ in the electrostatic approximation and in a homogeneous environment, whereas the experiment is performed on large antennas placed on a substrate.

(13) Pellarin, M.; Ramade, J.; Rye, J. M.; Bonnet, C.; Broyer, M.; Lebeault, M.-A.; Lermé, J.; Marguet, S.; Navarro, J. R.; Cottancin, E. Fano Transparency in Rounded Nanocube Dimers Induced by Gap Plasmon Coupling. ACS Nano 2016, 10, 11266-11279.

(14) Ortega-Arroyo, J.; Kukura, P. Interferometric scattering microscopy (iSCAT): new frontiers in ultrafast and ultrasensitive optical microscopy. Phys. Chem. Chem. Phys. 2012, 14, 15625.

(15) Young, G.; et al. Quantitative mass imaging of single biological macromolecules. Science 2018, 360, 423-427.

(16) Avci, O.; Adato, R.; Ozkumur, A. Y.; Ünlü, M. S. Physical modeling of interference enhanced imaging and characterization of single nanoparticles. Opt. Express 2016, 24, 6094.

(17) Sevenler, D.; Avci, O.; Ünlü, M. S. Quantitative interferometric reflectance imaging for the detection and measurement of biological nanoparticles. Biomed. Opt. Express 2017, 8, 2976.

(18) Anderson, L. J. E.; Mayer, K. M.; Fraleigh, R. D.; Yang, Y.; Lee, S.; Hafner, J. H. Quantitative Measurements of Individual Gold Nanoparticle Scattering Cross Sections. J. Phys. Chem. C 2010, 114, $11127-11132$.

(19) Anderson et al. ${ }^{18}$ propose to calibrate the scattering intensity measured under DF illumination using spheres of known size, whose $\sigma_{\text {sca }}$ is known analytically; however, such procedure is accurate only when the angular distribution of the power scattered by the measured $\mathrm{NanO}$ is close to the sphere distribution and must be repeated whenever the experimental setup is modified.

(20) Lukosz, W.; Kunz, R. Fluorescence lifetime of magnetic and electric dipoles near a dielectric interface. Opt. Commun. 1977, 20, 195-199.

(21) Lukosz, W.; Kunz, R. E. Light emission by magnetic and electric dipoles close to a plane interface. I. Total radiated power. J. Opt. Soc. Am. 1977, 67, 1607-1615.
(22) Lukosz, W.; Kunz, R. E. Light emission by magnetic and electric dipoles close to a plane dielectric interface. II. Radiation patterns of perpendicular oriented dipoles. J. Opt. Soc. Am. 1977, 67, $1615-1619$.

(23) Lukosz, W. Light emission by magnetic and electric dipoles close to a plane dielectric interface. III. Radiation patterns of dipoles with arbitrary orientation. J. Opt. Soc. Am. 1979, 69, 1495-1503.

(24) Johnson, P. B.; Christy, R. W. Optical constants of noble metals. Phys. Rev. B 1972, 6, 4370-4379.

(25) McPeak, K. M.; Jayanti, S. V.; Kress, S. J. P.; Meyer, S.; Iotti, S.; Rossinelli, A.; Norris, D. J. Plasmonic Films Can Easily Be Better: Rules and Recipes. ACS Photonics 2015, 2, 326-333.

(26) Olmon, R. L.; Slovick, B.; Johnson, T. W.; Shelton, D.; Oh, S.H.; Boreman, G. D.; Raschke, M. B. Optical dielectric function of gold. Phys. Rev. B: Condens. Matter Mater. Phys. 2012, 86, 235147.

(27) Payne, L.; Langbein, W.; Borri, P. Wide-Field Imaging of Single-Nanoparticle Extinction with Sub-nm ${ }^{2}$ Sensitivity. Phys. Rev. Appl. 2018, 9, 034006.

(28) Payne, L.; Zilli, A.; Wang, Y.; Langbein, W.; Borri, P. Quantitative high-throughput optical sizing of individual colloidal nanoparticles by wide-field imaging extinction microscopy. Proc. SPIE 10892, Colloidal Nanoparticles for Biomedical Applications XIV, 2019.

(29) Masia, F.; Langbein, W.; Borri, P. Measurement of the dynamics of plasmons inside individual gold nanoparticles using a femtosecond phase-resolved microscope. Phys. Rev. B: Condens. Matter Mater. Phys. 2012, 85, 235403.

(30) Muskens, O. L.; Billaud, P.; Broyer, M.; Del Fatti, N.; Vallée, F. Optical extinction spectrum of a single metal nanoparticle: Quantitative characterization of a particle and of its local environment. Phys. Rev. B: Condens. Matter Mater. Phys. 2008, 78, 205410.

(31) Berciaud, S.; Cognet, L.; Tamarat, P.; Lounis, B. Observation of Intrinsic Size Effects in the Optical Response of Individual Gold Nanoparticles. Nano Lett. 2005, 5, 515-518.

(32) Kawabata, A.; Kubo, R. Electronic Properties of Fine Metallic Particles. II. Plasma Resonance Absorption. J. Phys. Soc. Jpn. 1966, 21, $1765-1772$.

(33) Huang, X.; Neretina, S.; El-Sayed, M. A. Gold Nanorods: From Synthesis and Properties to Biological and Biomedical Applications. Adv. Mater. 2009, 21, 4880-4910.

(34) Goris, B.; Bals, S.; Van den Broek, W.; Carbó-Argibay, E.; Gómez-Graña, S.; Liz-Marzán, L. M.; Van Tendeloo, G. Atomic-scale determination of surface facets in gold nanorods. Nat. Mater. 2012, $11,930-935$

(35) Kekicheff, P.; Spalla, O. Refractive Index of Thin Aqueous Films Confined between Two Hydrophobic Surfaces. Langmuir 1994, $10,1584-1591$.

(36) Gómez-Graña, S.; Hubert, F.; Testard, F.; Guerrero-Martínez, A.; Grillo, I.; Liz-Marzán, L. M.; Spalla, O. Surfactant (Bi)Layers on Gold Nanorods. Langmuir 2012, 28, 1453-1459.

(37) Foerster, B.; Joplin, A.; Kaefer, K.; Celiksoy, S.; Link, S.; Sönnichsen, C. Chemical Interface Damping Depends on Electrons Reaching the Surface. ACS Nano 2017, 11, 2886-2893.

(38) Muskens, O. L.; Bachelier, G.; Fatti, N. D.; Vallée, F.; Brioude, A.; Jiang, X.; Pileni, M.-P. Quantitative Absorption Spectroscopy of a Single Gold Nanorod. J. Phys. Chem. C 2008, 112, 8917-8921.

(39) Davletshin, Y. R.; Lombardi, A.; Cardinal, M. F.; Juvé, V.; Crut, A.; Maioli, P.; Liz-Marzán, L. M.; Vallée, F.; Fatti, N. D.; Kumaradas, J. C. A Quantitative Study of the Environmental Effects on the Optical Response of Gold Nanorods. ACS Nano 2012, 6, 8183-8193.

(40) Lombardi, A.; Loumaigne, M.; Crut, A.; Maioli, P.; Del Fatti, N.; Vallée, F.; Spuch-Calvar, M.; Burgin, J.; Majimel, J.; TréguerDelapierre, M. Surface Plasmon Resonance Properties of Single Elongated Nano-objects: Gold Nanobipyramids and Nanorods. Langmuir 2012, 28, 9027-9033.

(41) Payne, L.; Langbein, W.; Borri, P. Extinction Suite plug-in for ImageJ. http://langsrv.astro.cf.ac.uk/Crosssection/Crosssection.html.

(42) Payne, L.; Langbein, W.; Borri, P. Polarization-resolved extinction and scattering cross-sections of individual gold nano- 
particles measured by wide-field microscopy on a large ensemble. Appl. Phys. Lett. 2013, 102, 131107.

(43) Payne, L.; Zoriniants, G.; Masia, F.; Arkill, K. P.; Verkade, P.; Rowles, D.; Langbein, W.; Borri, P. Optical micro-spectroscopy of single metallic nanoparticles: Quantitative extinction and transient resonant four-wave mixing. Faraday Discuss. 2015, 184, 305-320.

(44) Jones, S. H.; King, M. D.; Ward, A. D. Determining the unique refractive index properties of solid polystyrene aerosol using broadband Mie scattering from optically trapped beads. Phys. Chem. Chem. Phys. 2013, 15, 20735.

(45) Christensen, T.; Yan, W.; Jauho, A.-P.; Soljačić, M.; Mortensen, N. A. Quantum Corrections in Nanoplasmonics: Shape, Scale, and Material. Phys. Rev. Lett. 2017, 118, 157402.

(46) Zilli, A.; Langbein, W.; Borri, P. Quantitative measurement of the optical cross-sections of single nano-objects by correlative transmission and scattering micro-spectroscopy. Cardiff University; 2019, DOI: $10.17035 /$ d.2018.0064868399. 